\title{
Transport near a quantum critical point in $\mathrm{BaFe}_{2}\left(\mathrm{As}_{1-x} \mathrm{P}_{x}\right)_{2}$
}

\author{
James G. Analytis ${ }^{1,2,3,4 \star}$, H-H. Kuo ${ }^{1,5}$, Ross D. McDonald ${ }^{6}$, Mark Wartenbe ${ }^{3}$, P. M. C. Rourke ${ }^{7}$, \\ N. E. Hussey ${ }^{7,8}$ and I. R. Fisher ${ }^{1,2}$
}

\begin{abstract}
The physics of quantum critical phase transitions connects to some of the most difficult problems in condensed matter physics, including metal-insulator transitions, frustrated magnetism and high-temperature superconductivity. Near a quantum critical point, a new kind of metal emerges, the thermodynamic and transport properties of which do not fit into the unified phenomenology for conventional metals-the Landau Fermi-liquid theory-characterized by a low-temperature limiting $T$-linear specific heat and a $T^{2}$ resistivity'. Studying the evolution of the temperature dependence of these observables as a function of a control parameter leads to the identification of both the presence and the nature of the quantum phase transition in candidate systems. In this study we measure the transport properties of $\mathrm{BaFe}_{2}\left(\mathrm{As}_{1-x} \mathrm{P}_{x}\right)_{2}$ below the critical temperature $T_{c}$ by suppressing superconductivity with high magnetic fields. At sufficiently low temperatures, the resistivity of all compositions $(x \geqslant 0.31)$ crosses over from a linear to a quadratic temperature dependence, consistent with a low-temperature Fermi-liquid ground state. As compositions with optimal $T_{c}$ are approached from the overdoped side, this crossover becomes steeper, consistent with models of quantum criticality where the effective Fermi temperature $T_{F}$ goes to zero.
\end{abstract}

The iron-based superconductors are part of a family of unconventional superconductors that exhibit several competing orders. The parent material $\mathrm{BaFe}_{2} \mathrm{As}_{2}$ is a tetragonal paramagnet at high temperature and becomes an orthorhombic metallic antiferromagnet at $\sim 140 \mathrm{~K}$ (ref. 2). As the material is electron doped, hole doped or isovalently substituted this transition is rapidly suppressed, giving rise to superconductivity. In this work, we attempt to understand the nature of the low- $T$ metallic state of the Fe-based superconductor $\mathrm{BaFe}_{2}\left(\mathrm{As}_{1-x} \mathrm{P}_{x}\right)_{2}$ by suppressing the superconductivity in a high magnetic field. Even though $\mathrm{BaFe}_{2}\left(\mathrm{As}_{1-x} \mathrm{P}_{x}\right)_{2}$ is isovalently substituted, we will describe the chemical composition-temperature $(x-T)$ phase diagram using language commonly applied to electron/hole-doped compounds, namely 'underdoped' refers to materials that exhibit a structural/magnetic instability, and 'overdoped' for paramagnetic compounds that do not. For this material, the maximum $T_{\mathrm{c}}$ (optimal doping) occurs at $x=0.30$.

$\mathrm{BaFe}_{2}\left(\mathrm{As}_{1-x} \mathrm{P}_{x}\right)_{2}$ is a multi-band compound with both electronand hole-like carriers and the magnetoresistance is therefore a sum of contributions from all Fermi surfaces. In Fig. 1 we illustrate the magnetoresistance as a function of temperature and field for a range of compositions from $x=0.31$ to $x=0.73$ and $T_{\mathrm{c}}$ spanning $29.5 \mathrm{~K}$ to $0 \mathrm{~K}$. For all temperatures measured, a quadratic magnetoresistance fit captures most of the data and the intercept $\rho_{0, T}$ is extrapolated (shown by black lines in Fig. 1). At low fields, this fit deviates from the quadratic dependence in the near-optimally doped samples, even at temperatures $T>T_{\mathrm{c}}$, although the deviation ostensibly disappears at sufficiently high $T$ (see Supplementary Section A). This may be due to a complex balance of mobilities between electron and hole pockets or due to superconducting fluctuations persisting at $T>T_{\mathrm{c}}$, as seen in cuprate materials ${ }^{3,4}$. Thus, $\rho_{0, T}$ is slightly overestimated, causing an offset in the zero-field resistance estimate $\left.\delta_{\mathrm{R}} \equiv\left(\rho_{\text {exptl }}-\rho_{\text {fit }}\right)\right|_{0, T}$. Within the present data set, this offset is of the order $\sim 10^{-2} \rho / \rho_{300}$. We proceed assuming that $\delta_{\mathrm{R}}$ has a negligible effect on the evolution of $\rho_{0, T}$ with $T$, and discuss the consequences of this assumption in Supplementary Section B. In the simplest case of an ordinary metal, the magnetoresistance of a multi-band system is expected to be $\propto B^{2}$ at low magnetic fields, but for an unequal number of electrons and holes the resistivity will eventually saturate ${ }^{5}$. If a material is perfectly compensated, however, the magnetoresistance will remain $B^{2}$ up to very high fields ${ }^{6}$. The compound $\mathrm{BaFe}_{2}\left(\mathrm{As}_{1-x} \mathrm{P}_{x}\right)_{2}$ is isovalently substituted, forcing the system to remain compensated ${ }^{6,7}$ as a function of $x$. The magnetoresistance thus exhibits a $B^{2}$ dependence over a wide field range, consistent with our observations. It should be noted that recent photoemission work ${ }^{6}$ has observed a slight hole doping with $\mathrm{P}$ substitution at the surface near optimal $\mathrm{P}$ compositions (but returning to perfectly compensated in the overdoped region). However, the ratio $n_{\mathrm{h}} / n_{\mathrm{e}}$ remains close, implying that $B^{2}$ resistivity is appropriate for values of the magnetoresistance up to $\rho(B) \sim$ $10 \times \rho_{0, T}$ for optimally doped samples (where the affect is greatest). In Fig. 1 the largest magnetoresistance we observe at optimal compositions occurs at $4 \mathrm{~K}$ and $\rho(B)<1.2 \times \rho_{0, T}$, if $\rho_{0, T}$ is taken as the $B=0$ intercept, putting the present data far from the saturation regime. We therefore analyse this data with the quadratic functional form with some confidence of its validity. As a further caveat, it is important to point out that an established understanding of magnetoresistance near a quantum critical metal does not at present exist, and this fact may compromise our model of magnetoresistance (although several treatments exist $\left.{ }^{8}\right)$. Nevertheless, our data suggest that a $B^{2}$ law is

\footnotetext{
${ }^{1}$ Stanford Institute for Materials and Energy Sciences, SLAC National Accelerator Laboratory, 2575 Sand Hill Road, Menlo Park, California 94025, USA, ${ }^{2}$ Geballe Laboratory for Advanced Materials and Department of Applied Physics, Stanford University, California 94305, USA, ${ }^{3}$ National High Magnetic Field Laboratory, Florida State University, Tallahassee, Florida 32310, USA, ${ }^{4}$ Department of Physics, University of California, Berkeley, California 94720 , USA, ${ }^{5}$ Department of Materials Science and Engineering, Stanford University, California 94305, USA, ${ }^{6}$ National High Magnetic Field Laboratory, Los Alamos National Laboratory, Los Alamos, New Mexico 87545, USA, ${ }^{7} \mathrm{H}$. H. Wills Physics Laboratory, University of Bristol, Tyndall Avenue, Bristol BS8 $1 T L$, UK, ${ }^{8}$ High Field Magnet Laboratory, Institute of Molecules and Materials, Radboud University Nijmegen, Toernooiveld 7, 6525 ED Nijmegen, Netherlands. *e-mail: analytis@berkeley.edu
} 


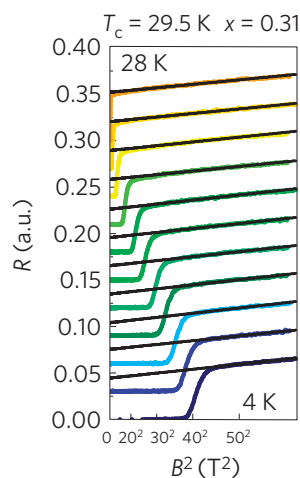

$B^{2}\left(T^{2}\right)$

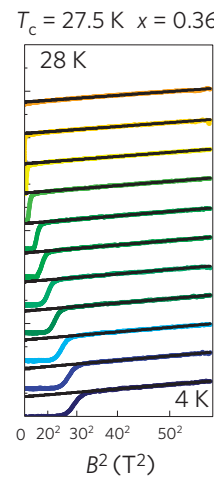

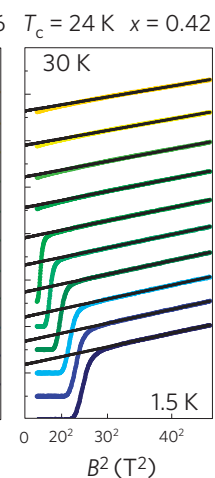

$B^{2}\left(T^{2}\right)$

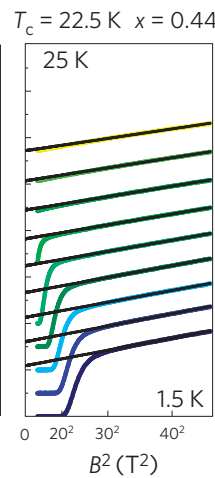

$B^{2}\left(T^{2}\right)$

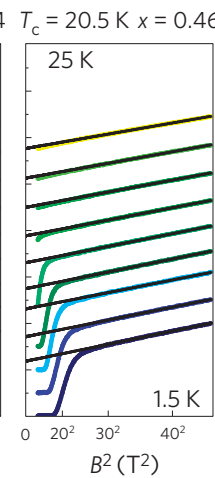

$B^{2}\left(T^{2}\right)$
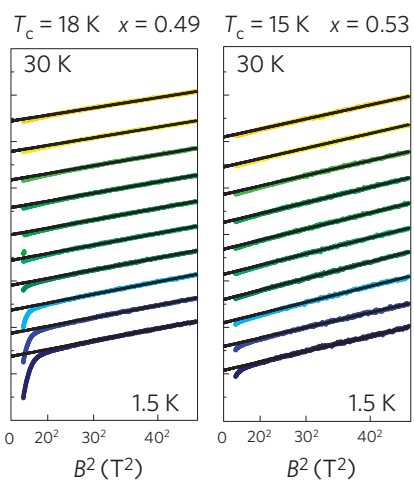

Figure 1 | Magnetic field dependence of the magnetoresistance for overdoped $\mathbf{B a F e}_{2}\left(\mathbf{A s}_{\mathbf{1}_{-x}} \mathbf{P}_{\mathbf{x}}\right)_{2}$. Each of the seven samples has a distinct $T_{\mathrm{C}}$. Each different coloured curve has been offset for clarity and corresponds to a different temperature in the range indicated; raw data curves are in Supplementary Section $A$. The black lines are fits to a standard magnetoresistance model $\rho(B)=\rho_{0, T}+A_{B} B^{2}$.
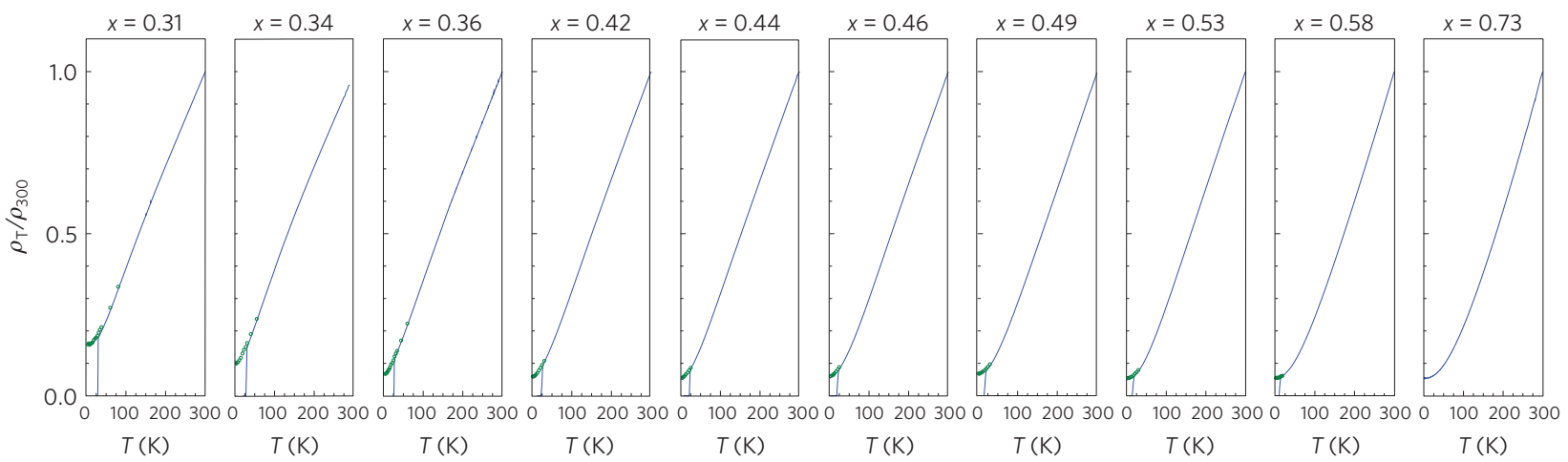

Figure 2 | The zero-field temperature dependence of the resistivity at the indicated composition. Each curve has been normalized to the room-temperature value $\rho_{T} / \rho_{300}$ in blue, together with the zero-field intercept extracted from the corresponding high-field magnetoresistance shown in Fig. 1 in green.

valid, at least empirically, for compounds in the overdoped part of the pnictide phase diagram.

In Fig. 2 we show the zero-field normalized resistivity cooling curves of these materials in blue and the values of $\rho_{0, T}$ extrapolated from the data in Fig. 2 in green-the latter connects smoothly to the former such that analysis of the power law is possible at $T<T_{\text {c }}$. There are broadly two schools of thought as to how this power law should be analysed in correlated electron systems. Recent studies on the cuprates ${ }^{9}$, and on pnictide and organic superconductors ${ }^{10}$ have suggested a transport law that combines linear and quadratic components

$$
\rho=\rho_{0,0}+A T+B T^{2}
$$

However, in heavy-fermion materials such as $\mathrm{CePd}_{2} \mathrm{Si}_{2}$ (refs 11 , 12 ) it is found that a single power law $n$ captures the transport behaviour, accounting for a crossover from Fermi-liquid to quantum critical regimes as some critical parameter is varied, such as doping or magnetic field. In its most general form the transport will look like

$$
\rho=\rho_{0,0}+A T^{n}
$$

Taking the derivative provides a simple way to distinguish equation (1) from equation (2), giving a finite (negligible) intercept in the former (latter). In Fig. 3a,b we illustrate this derivative for all of the samples considered using the normal-state data shown in Fig. 2. In all cases, we observe (within experimental error) a zero intercept, suggesting that equation (2) is best able to describe the data for $\mathrm{BaFe}_{2}\left(\mathrm{As}_{1-x} \mathrm{P}_{x}\right)_{2}$. Furthermore, the $\mathrm{d} \rho / \mathrm{d} T$ curves are linear at low temperature, suggesting that all samples recover a $n=2$ power law expected of a Fermi liquid. As optimal doping is approached, the range in $T$ over which this applies steadily decreases. In addition, the slope of these curves $(=2 A)$ sharply peaks, or even diverges, as optimal doping is approached, as shown in Fig. 3c. In certain cuprate and heavyfermion superconductors, this behaviour has been interpreted as an indication of the proximity to a quantum critical point ${ }^{9,13,14}$ (QCP). In this context, the cusp seen in $\mathrm{d} \rho / \mathrm{d} T$ perhaps marks the crossing of a border in the $x-T$ phase diagram, above which quantum critical fluctuations dominate.

The presence of a QCP in the P-substituted phase diagram has been suggested by certain thermodynamic measurements, ${ }^{75-17}$. In the traditional approach to quantum criticality ${ }^{18}$, the effective dimensionality $d_{\text {eff }}$ is defined as the sum of the spatial dimension $d$ and the dynamical exponent $z$, which characterizes the temporal fluctuations. In quasi-two-dimensional systems near an antiferromagnetic (AFM) critical point, $d=2, z=2$ and $d_{\text {eff }}(=4)$ is at the upper critical dimension where one would expect the specific heat divided by temperature to vary as $\left[C_{0}-C_{1} \ln \left(x-x_{\mathrm{c}}\right)\right]$ (ref. 19). Comparing our study to previous determinations ${ }^{17}$ of $m^{* 2}$, we observe that our data are consistent with this model if the electron-electron scattering is assumed to be proportional to the mass enhancement, namely that the Kadowaki-Woods ratio (KWR) holds ${ }^{20}$. It has been shown that the average mass enhancement (of one of the Fermi surfaces) quantitatively agrees with the mass enhancement estimated by comparing the Sommerfeld coefficient $\gamma$ to the densityfunctional calculated value $\gamma_{0}=6.94 \mathrm{~mJ} \mathrm{~mol}^{-1} \mathrm{~K}^{-2}$, where $\gamma$ was determined from the superconducting heat capacity jump $\Delta C / T_{\mathrm{c}}$ (ref. 17). Using this renormalization of the band mass $m^{*} / m_{\mathrm{b}}$, we 


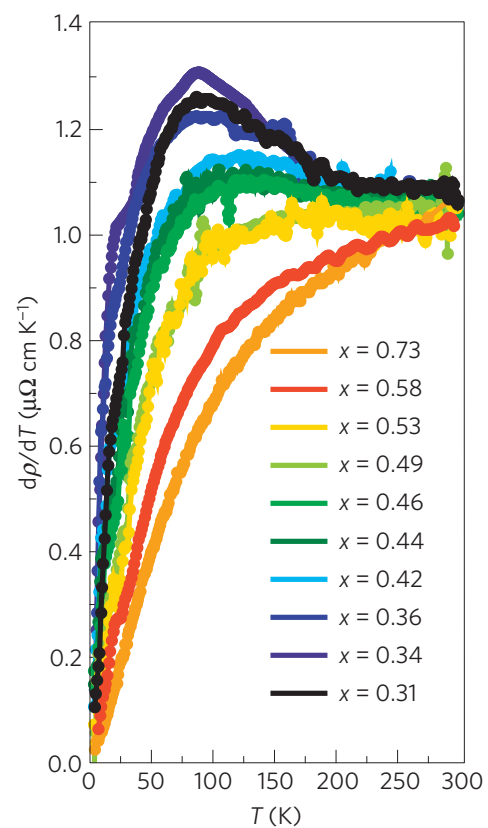

b

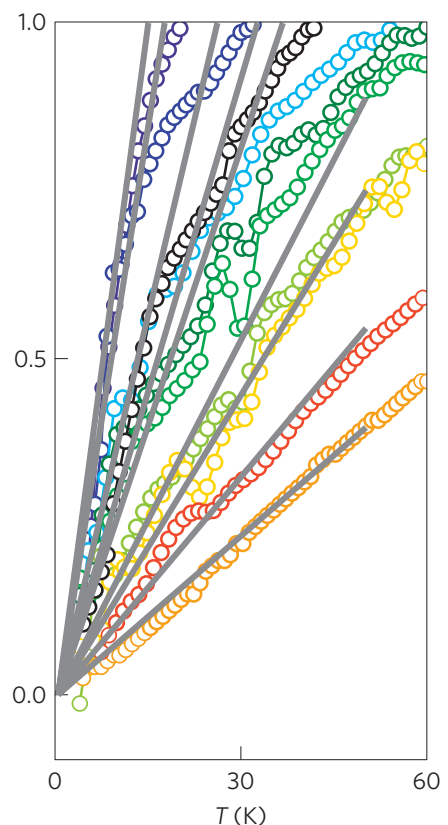

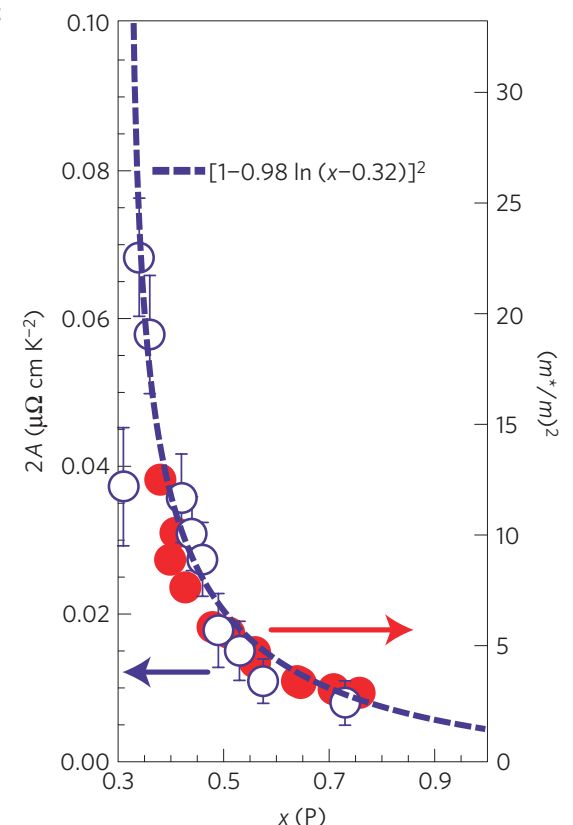

Figure 3 | Derivative of the resistivity interpolated over the full temperature range. a, The derivative of the resistivity for all samples in Fig. 2, including data from zero-field and high-field experiments, interpolated to form differentiable curves. $\mathbf{b}$, An enlargement of the data shown in a with linear fits to the low- $T$ behaviour (grey lines). c, The pronounced increase in the value $2 A$ (open circles) at compositions between $x=0.73$ and 0.37 , suggests the proximity of a QCP below the superconducting dome. The data are consistent with the KWR (ref. 20) $A / m^{* 2}=$ constant, as can be seen by comparison with previous determinations (red circles) of the effective mass $m^{*}$ (refs 15-17). The line is a fit to a phenomenological divergence of the effective mass near a QCP (ref. 19) $\left[C_{0}-C_{1} \ln \left(x-x_{c}\right)\right]^{2}$, with $x_{c}=0.32$. Error bars have been determined using the standard deviation of the data from the least-squares fit.

estimate that the $\mathrm{KWR} A / \gamma^{2}$ ranges from $\approx 7 \mu \Omega \mathrm{cm} \mathrm{mol}^{2} \mathrm{~K}^{2} \mathrm{~J}^{-2}$ at $x=0.34$, to $\approx 15 \mu \Omega \mathrm{cm} \mathrm{mol}^{2} \mathrm{~K}^{2} \mathrm{~J}^{-2}$ for our most overdoped sample $x=0.73$. The factor of two change could arise from the discrepancy in estimating $m^{*}$ from the heat capacity jump versus the de Haas-van Alphen mass in ref. 17. These values agree with other correlated electron materials, specifically heavy-fermion compounds that have $A / \gamma^{2} \sim 10 \mu \Omega \mathrm{cm} \mathrm{mol}^{2} \mathrm{~K}^{2} \mathrm{~J}^{-2}$ (refs 20-22). We subsequently fit our data to a phenomenological critical power law $^{19} A \propto\left[C_{0}-C_{1} \ln \left(x-x_{\mathrm{c}}\right)\right]^{2}$ over $x \geq 0.34$. If we fix $x_{\mathrm{c}}=0.32$ and $C_{0}=1$, the fit yields $C_{1}=0.98$. This curve is shown as a dashed line in Fig. 3c. The fit is sensitive to the choice of $x_{c}$, and choosing $x_{c}=0.3$ yields the same values as ref. 17. The difference between the present determination of $x_{\mathrm{c}}$ and earlier works ${ }^{15-17}$ may be due to the effect of finite magnetic field ${ }^{13}$. On the other hand, it may be due to the complicated nature of interpreting transport at compositions near the AFM transition (See Supplementary Section C), or deviations of the magnetoresistance from conventional behaviour near a QCP (ref. 8). In addition, even though $\mathrm{BaFe}_{2}\left(\mathrm{As}_{1-x} \mathrm{P}_{x}\right)_{2}$ is the cleanest of the superconducting materials with the $\mathrm{BaFe}_{2} \mathrm{As}_{2}$ structure ${ }^{23}$, it is possible that disorder also affects the exact determination of $x_{c}$.

We illustrate the evolution of the power law $n$ as a function of temperature and doping in Fig. 4 . We fit $\mathrm{d} \rho / \mathrm{d} T$ with a sliding temperature window to the equation $\mathrm{d} \rho / \mathrm{d} T=n A T^{n-1}$, with a window width $\Delta T=7 \mathrm{~K}$. From this we extract the average power law $n(T)$, where the mid-point of the window is taken as the temperature, shown in Fig. 4. For all compositions considered, $n \rightarrow 2$ at low $T$, consistent with the linear slope observed in $\mathrm{d} \rho / \mathrm{d} T$, Fig. $3 \mathrm{~b}$. The crossover to $n=2$ may be interpreted as the re-entrance to Fermi-liquid behaviour (marked $\left(T_{\mathrm{FL}}\right)$ in Fig. 4). Compositions in the range $0.31<x<0.34$, where Fig. $3 c$ suggests $2 A$ diverges, are indicated by a grey line.

In marked contrast to the situation found for hole-doped cuprates, where the $T$-linear resistivity remains robust over an extended range of doping $9, \mathrm{BaFe}_{2}\left(\mathrm{As}_{1-x} \mathrm{P}_{x}\right)_{2}$ is found to exhibit behaviour associated with a more conventional picture of quantum

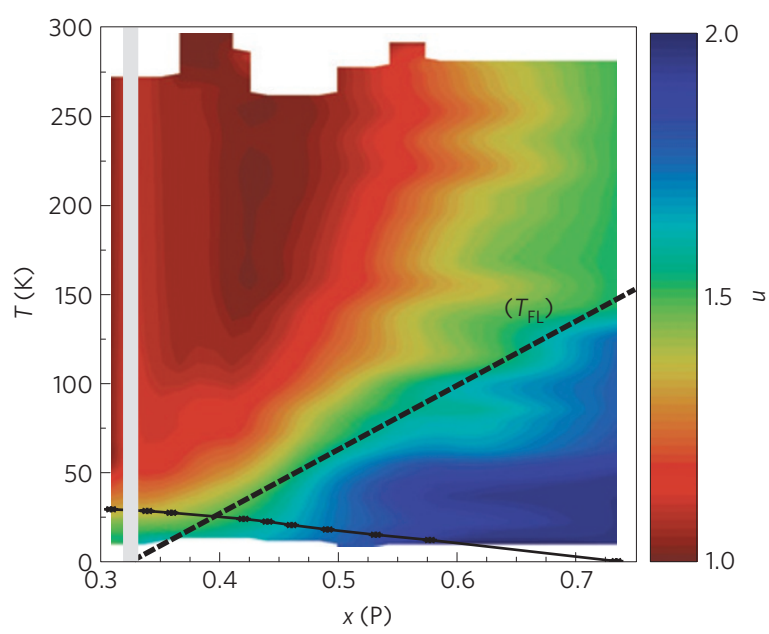

Figure 4 | Colour plot of the power-law dependence $n$ as a function of temperature and doping. The data have been interpolated over the discrete dopings measured. The black dashed line is a guide to the eye, indicating the region of the phase diagram that can be interpreted as the re-entrance of Fermi-liquid-like behaviour $\left(T_{\mathrm{FL}}\right)$. Compositions where $2 \mathrm{~A}$ is thought to diverge (Fig. $3 \mathrm{C}$ ) are indicated by the grey line. The black data points connected by a solid line are the superconducting $T_{\mathrm{c}}$ at that composition.

criticality in which $\rho(T)$ always crosses over from a $T$-linear to a $T^{2}$ dependence below a temperature scale that diminishes on approach to the QCP. In accordance with this picture, the coefficient of the $T^{2}$ resistivity is also found to diverge as the QCP is approached. Although there is at present no accepted theory explaining the origin of $T$-linear resistivity in quantum critical metals, a common point of view is that it is associated with Fermi-surface 'hotspots'-regions of the Fermi surface with strongly enhanced scattering. Such regions are thought to dominate 
the transport of many strongly correlated metals ${ }^{24}$, particularly as disorder increases ${ }^{12}$, accounting for the strong dependence of the scattering on $T$. Away from optimal doping, the iron-based superconductors order anti-ferromagentically, and hotspots on electron and hole Fermi surfaces ${ }^{25}$ are therefore expected. Our data can be broadly understood in this context-as an AFM QCP is approached from the overdoped side, the Fermi-surface hotspots become increasingly 'hotter', sharply changing the nature of the transport as a function of temperature. However, why it is that the resistivity is strictly $T$-linear and why this dependence is common to so many metals near a QCP remains an open question.

\section{Methods}

Single crystals of $\mathrm{BaFe}_{2}\left(\mathrm{As}_{1-x} \mathrm{P}_{x}\right)_{2}$ were grown by a self-flux method described elsewhere ${ }^{23}$. High magnetic fields were made available at the NHMFL, Tallahassee ( $45 \mathrm{~T}$, static) and at Los Alamos National Labs (65 T pulsed). In the presence of a magnetic field, the stationary states are quantized into Landau levels, which are separated by the cyclotron energy $\hbar \omega_{\text {cyc }}$. When a material is superconducting, this energy scale competes with the nucleation of Cooper pairs defining an upper critical field $H_{\mathrm{c} 2}$, which depends on $1 / \xi^{2}$, where $\xi$ is the superconducting coherence length. Beyond $H_{\mathrm{c} 2}$ the system is considered to be in the 'normal metal' state, exhibiting metallic magnetoresistance. Samples with a $T_{\mathrm{c}} \leq 24 \mathrm{~K}$ were measured in the hybrid magnet in Tallahassee; samples with $T_{\mathrm{c}} \geq 27.5 \mathrm{~K}$ were measured in pulsed fields in Los Alamos.

Received 28 May 2013; accepted 9 December 2013; published online 19 January 2014

\section{References}

1. Landau, L. The theory of a Fermi liquid. J. Exp. Theoret. Phys. 6, 920-925 (1957).

2. Rotter, M., Hieke, C. \& Johrendt, D. Different response of the crystal structure to isoelectronic doping in $\mathrm{BaFe}_{2}\left(\mathrm{As}_{1-x} \mathrm{P}_{x}\right)_{2}$ and $\left(\mathrm{Ba}_{1-x} \mathrm{Sr}_{x}\right) \mathrm{Fe}_{2} \mathrm{As}_{2}$. Phys. Rev. $B$ 82, 014513 (2010).

3. Rourke, P. M. C. et al. Phase-fluctuating superconductivity in overdoped $\mathrm{La}_{2-x} \mathrm{Sr}_{x} \mathrm{CuO}_{4}$. Nature Phys. 7, 455-458 (2011).

4. Rullier-Albenque, F., Alloul, H. \& Rikken, G. High-field studies of superconducting fluctuations in high- $T_{\mathrm{c}}$ cuprates: Evidence for a small gap distinct from the large pseudogap. Phys. Rev. B 84, 014522 (2011).

5. Pippard, A. B. Magnetoresistance in Metals 1st edn (Cambridge Univ. Press, 2009).

6. Ye, Z. R. et al. Doping dependence of the electronic structure in phosphorus-doped ferropnictide superconductor $\mathrm{BaFe}_{2}\left(\mathrm{As}_{1-x} \mathrm{P}_{x}\right)_{2}$ studied by angle-resolved photoemission spectroscopy. Phys. Rev. B 86, 035136 (2012).

7. Kasahara, S. et al. Evolution from non-Fermi- to Fermi-liquid transport via isovalent doping in $\mathrm{BaFe}_{2}\left(\mathrm{As}_{1-x} \mathrm{P}_{x}\right)_{2}$ superconductors. Phys. Rev. B 81, 184519 (2010).

8. Fenton, J. \& Schofield, A. J. Breakdown of weak-field magnetotransport at a metallic quantum critical point. Phys. Rev. Lett. 95, 247201 (2005).

9. Cooper, R. A. et al. Anomalous criticality in the electrical resistivity of $\mathrm{La}_{2-x} \mathrm{Sr}_{x} \mathrm{CuO}_{4}$. Science 323, 603-607 (2009).

10. Doiron-Leyraud, N. et al. Correlation between linear resistivity and $T_{\mathrm{c}}$ in the Bechgaard salts and the pnictide superconductor $\mathrm{Ba}\left(\mathrm{Fe}_{1-x} \mathrm{Co}_{x}\right) 2 \mathrm{As}_{2}$. Phys. Rev. B 80, 214531 (2009).
11. Custers, J. et al. The break-up of heavy electrons at a quantum critical point. Nature 424, 524-527 (2003).

12. Rosch, A. Interplay of disorder and spin fluctuations in the resistivity near a quantum critical point. Phys. Rev. Lett. 82, 4280-4283 (1999).

13. Butch, N. P., Jin, K., Kirshenbaum, K., Greene, R. L. \& Paglione, J. Quantum critical scaling at the edge of Fermi liquid stability in a cuprate superconductor. Proc. Natl Acad. Sci. USA 109, 8440-8444 (2012).

14. Taillefer, L. Scattering and pairing in cuprate superconductors. Annu. Rev. Condens. Matter Phys. 1, 51-70 (2010).

15. Hashimoto, K. et al. A sharp peak of the zero-temperature penetration depth at optimal composition in $\mathrm{BaFe}_{2}\left(\mathrm{As}_{1-x} \mathrm{P}_{x}\right)_{2}$. Science 336, 1554-1557 (2012).

16. Shishido, H. et al. Evolution of the Fermi surface of $\mathrm{BaFe}_{2}\left(\mathrm{As}_{1-x} \mathrm{P}_{x}\right)_{2}$ on entering the superconducting dome. Phys. Rev. Lett. 104, 057008 (2010).

17. Walmsley, P. et al. Quasiparticle mass enhancement close to the quantum critical point in $\mathrm{BaFe}_{2}\left(\mathrm{As}_{1-x} \mathrm{P}_{x}\right)_{2}$. Phys. Rev. Lett. 110, 257002 (2013).

18. Hertz, J. A. Quantum critical phenomena. Phys. Rev. B 14, 1165-1184 (1976).

19. Abrahams, E. \& Si, Q. Quantum criticality in the iron pnictides and chalcogenides. J. Phys. Condens. Matter 23, 223201 (2011).

20. Kadowaki, K. \& Woods, S. Universal relationship of the resistivity and specific heat in heavy-fermion compounds. Solid State Commun. 58, 507-509 (1986).

21. Hussey, N. E. Non-generality of the KadowakiWoods ratio in correlated oxides. J. Phys. Soc. Jpn 74, 1107-1110 (2005).

22. Jacko, A. C., Firestad, J. O. \& Powell, B. J. A unified explanation of the KadowakiWoods ratio in strongly correlated metals. Nature Phys. 5, 422-425 (2009).

23. Analytis, J. G., Chu, J., McDonald, R. D., Riggs, S. C. \& Fisher, I. R. Enhanced Fermi-surface nesting in superconducting $\mathrm{BaFe}_{2}\left(\mathrm{As}_{1-x} \mathrm{P}_{x}\right)_{2}$ revealed by the de Haas van Alphen effect. Phys. Rev. Lett. 105, 207004 (2010).

24. Bruin, J. a. N., Sakai, H., Perry, R. S. \& Mackenzie, A. P. Similarity of scattering rates in metals showing t-linear resistivity. Science 339, 804-807 (2013).

25. Fernandes, R. M., Abrahams, E. \& Schmalian, J. Anisotropic in-plane resistivity in the nematic phase of the iron pnictides. Phys. Rev. Lett. 107, 217002 (2011).

\section{Acknowledgements}

H-H.K., J.G.A. and I.R.F. acknowledge support of the US DOE, Office of Basic Energy Sciences under contract DE-AC02-76SF00515. J.G.A. would like to thank the NHMFL Visiting Scientist Program for valuable support while this data was taken. N.E.H. acknowledges a Royal Society Wolfson Research Merit Award and funding from the EPSRC (UK) grant EP/K016709/1. The National High Magnetic Field Laboratory is supported through NSF/DMR 1157490. R.D.M. acknowledges US DOE BES-'Science of 100 tesla'.

\section{Author contributions}

J.G.A. and I.R.F. conceived of this experiment. J.G.A., R.D.M., H.H.K., P.M.C.R. and M.W. performed the experiments. J.G.A., R.D.M., N.E.H. and I.R.F. performed the analysis. All authors contributed in writing the paper.

\section{Additional information}

Supplementary information is available in the online version of the paper. Reprints and permissions information is available online at www.nature.com/reprints. Correspondence and requests for materials should be addressed to J.G.A.

\section{Competing financial interests}

The authors declare no competing financial interests. 